\title{
Clinical profile and outcome of dengue among hospitalized children - a single centre prospective study
}

\author{
Srinivasa $\mathbf{K}^{1}$, Ajay $\mathbf{J}^{2}$, Manjunath G.A $\mathbf{A}^{3}$ \\ ${ }^{1}$ Dr. Srinivasa K, Associate Professor, ${ }^{2}$ Dr. Ajay J, Assistant Professor, ${ }^{3}$ Dr. Manjunath G.A., Professor and HOD, all \\ authors are affiliated with Department of Pediatrics, Navodaya Medical College Hospital and Research Institute, Raichur. \\ Karnataka, India.
}

Address for Correspondence: Dr. Srinivasa K, Email: drsriniks@gmail.com

\begin{abstract}
Introduction: Dengue is the most rapidly spreading mosquito-borne viral disease in the world.In India, there is increased proportion of Dengue cases with severe disease. Early recognition and prompt initiation of appropriate treatment are vital to reduce disease related morbidity and mortality. Objective: To study the clinical profile and outcome of hospitalized dengue fever cases in children during outbreaks. Methods: This is an observational prospective study conducted at tertiary care hospital. All children who presented with clinical features of dengue \& dengue serology positive children were included in the study. Results: A total of 185 children diagnosed to have dengue fever were admitted in the hospital during the study period. The cases were classified according to WHO protocol as Dengue without warning sign (48.1\%), Dengue with warning signs (27\%) and severe dengue (24.8\%). All the children had fever as the presenting complaint. The other predominant symptoms observed were vomiting (61.6\%), abdominal pain $(50.2 \%)$, rashes $(30.2 \%)$, myalgia (24.3\%), and bleeding manifestation (16.2\%). The other significant findings noted were Hepatomegaly (52.4\%), Ascites (47.01\%), and pleural effusion (41\%). Gallbladder wall thickness $(80.4 \%)$ was the most common radiological abnormality. Mortality in this study was $1.08 \%$. Conclusion: Children in the age group of 5-15 years were most commonly affected. Fever, Persistent vomiting, abdominal pain, Hepatomegaly, pleural effusion, were predominant presenting symptoms and signs. Early recognition, precise assessment and appropriate treatment with the help of WHO revised classification and management guidelines have reduced the mortality.
\end{abstract}

Keywords: Children, Dengue, Revised classification, Warning signs, WHO

\section{Introduction}

Dengue is the most rapidly spreading mosquito-borne viral disease in the world. In the last 50 years, incidence has increased 30-fold with increasing geographic expansion to new countries and in the present decade, from urban to rural settings. An estimated 50 million dengue infections occur annually and approximately 2.5 billion people live in dengue endemic countries [1]. In India, there is increased proportion of Dengue cases with severe disease.

The dengue epidemics in India are cyclical and are more frequent, expanding geographically into the rural areas and all forms of serotypes are circulating in the community [2]. Uncontrolled population growth, urbanization, inadequate waste water management, and

Manuscript received: $28^{\text {th }}$ January 2017

Reviewed: $6^{\text {th }}$ February 2017

Author Corrected: $15^{\text {th }}$ February 2017

Accepted for Publication: $21^{\text {st }}$ February 2017 lack of effective mosquito control have been implicated in the increased distribution and density of the vector and also the increased spread of the virus [3]. The manifestation of dengue fever vary from asymptomatic to severe dengue fever and differ from epidemic to epidemic with atypical manifestation. Early recognition of severe dengue infection and proper treatment is very important to reduce the morbidity and mortality.

WHO revised their guidelines in 2009 [4] and accordingly the clinical classification was revised as dengue without warning signs, dengue with warning signs, and severe dengue which was more appropriate and much easier to understand [5]. It also helped in identifying sick dengue patients easier for the clinicians than the traditional guidelines [6]. The warning signs in the revised classification were put forth to identify the severe dengue cases by health care professionals early 
during endemic and facilitated them for the need of admission and more intensive monitoring without the help of detailed laboratory workup [5,6]. Hospital based studies on the risk of shock and death in severe dengue in tropical Asian countries showed that the percentage of admitted cases developing shock, ranged from 9 to $60 \%$ and with in-hospital, case fatality rates ranging from 0.2 to over $9 \%$. Early recognition and prompt initiation of appropriate treatment are vital to reduce disease related morbidity and mortality. Additional data about the disease may leads to enactment or alteration in public health programs. Hence present study was undertaken to analyze the clinical profile and outcome of hospitalized dengue fever cases during outbreaks by adopting revised WHO guidelines 2009.

\section{Material and Methods}

This is an observational prospective study conducted at tertiary care hospital at Raichur, karnataka from June 2015 through November 2016. All patients who presented with symptoms of fever, body ache, vomiting, rashes, pain abdomen, headache and bleeding manifestation with confirmed dengue serology were included in the study.

A total of 185 children tested positive for dengue serology with clinical features of dengue were included in study. Children with negative Dengue serology were excluded from study. Informed consent was taken from parents/guardian. A detailed history and physical examination was done and findings were recorded. All suspected dengue patients were tested for both Dengue NS1 antigen test (within five days of fever) and Dengue $\mathrm{IgM} / \mathrm{IgG}$ rapid test (immunno-chromatographic test) after 5-6 days of fever. Laboratory investigations included complete hemogram, serum electrolytes, PT, APTT, liver function tests, renal function tests, chest X ray, and ultrasound abdomen. Cases tested positive were classified as per revised WHO classification as Dengue fever without warning signs, Dengue fever with warning signs, and Severe Dengue. The cases were treated according to WHO protocol. The mortality in terms of death was noted down. The data collected was analyzed, using chi-square test. The statistical package used was SPSS version.

\section{Results}

A total of 185 children diagnosed to have dengue fever were admitted in the hospital during the study period. Among them 122 were boys and 63 were girls with M: F ratio of 1.9:1.

Table-1: Age wise distribution of Dengue cases.

\begin{tabular}{|c|c|c|c|c|}
\hline Age (yrs) & $\begin{array}{c}\text { Dengue fever without } \\
\text { warning signs ( \%) }\end{array}$ & $\begin{array}{c}\text { Dengue fever with } \\
\text { warning signs ( \% ) }\end{array}$ & Severe Dengue (\%) & Total ( \% ) \\
\hline$<1$ & $2(2.2 \%)$ & $3(6 \%)$ & $2(4.3 \%)$ & $7(3.78 \%)$ \\
\hline $1-5$ & $11(12.3 \%)$ & $9(18 \%)$ & $3(6.5 \%)$ & $23(12.4 \%)$ \\
\hline $6-10$ & $39(43.8 \%)$ & $20(40 \%)$ & $16(34.7 \%)$ & $75(40.5 \%)$ \\
\hline$>10$ & $37(41.6 \%)$ & $18(36 \%)$ & $25(54.3 \%)$ & $80(43.2 \%)$ \\
\hline Total & $\mathbf{8 9}(\mathbf{4 8 . 1 \%})$ & $\mathbf{5 0}(\mathbf{2 7 \%})$ & $\mathbf{4 6}(\mathbf{2 4 . 8} \%)$ & $\mathbf{1 8 5}$ \\
\hline
\end{tabular}

From the above table the predominant age of presentation was 5-15 years. The mean age of presentation in this study was 8.2 years. Severe dengue was more commonly seen in children above 10 years $(54.3 \%)$ of age which is however statistically not significant.

From the above table Fever was the most common presenting symptom noted in all cases of dengue i.e. $100 \%$. Other complaints presented were vomiting (61.6\%), myalgia (24.3\%), Rashes (30.2\%), facial puffiness (49.1\%), flushing (35.5\%), conjunctival congestion (44.6\%), abdominal pain(50.2\%), bleeding manifestation (16.2\%), and joint pain (4.5\%). Abdominal pain, conjunctival congestion and facial puffiness were more common in Dengue with warning signs \& severe dengue group. Among bleeding manifestation, Malena and epistaxis were common symptoms noted along with petichae and gum bleeding. Erythematous macular Rashes with facial flushing were seen in $30.2 \%$ of children and in most of the cases it was maculopapular. Hepatomegaly was present in $86.9 \%$ in severe group and $74 \%$ in Dengue with warning signs group. It was statistically significant when compared with non-severe Dengue group ( $\mathrm{P}=0.000)$. Based on symptoms, signs \& investigations, $48.1 \%$ cases were classified as Dengue without warning signs, $27 \%$ as Dengue with 
Table-2: Clinical symptoms \& signs of Dengue in percentage.

\begin{tabular}{|c|c|c|c|c|}
\hline Clinical Profile & $\begin{array}{c}\text { Dengue without } \\
\text { warning signs (n= 89) }\end{array}$ & $\begin{array}{c}\text { Dengue with warning } \\
\text { signs (n= 50) }\end{array}$ & $\begin{array}{c}\text { Severe dengue } \\
(\mathbf{n = 4 6 )}\end{array}$ & Total (n= 185) \\
\hline Fever & $89(100 \%)$ & $50(100 \%)$ & $46(100 \%)$ & $185(100 \%)$ \\
\hline Headache & $21(23.5 \%)$ & $19(38 \%)$ & $20(43.4 \%)$ & $60(32.4 \%)$ \\
\hline Myalgia & $19(21.3 \%)$ & $14(28 \%)$ & $12(26 \%)$ & $45(24.3 \%)$ \\
\hline Vomiting & $40(44.9 \%)$ & $39(78 \%)$ & $35(76.1 \%)$ & $114(61.6 \%)$ \\
\hline Abdominal pain & $10(11.2 \%)$ & $41(82 \%)$ & $42(91.3 \%)$ & $93(50.2 \%)$ \\
\hline Facial puffiness & $19(21.3 \%)$ & $33(66 \%)$ & $39(84.7 \%)$ & $91(49.1 \%)$ \\
\hline Rash & $23(25.8 \%)$ & $17(34 \%)$ & $16(34.7 \%)$ & $56(30.2 \%)$ \\
\hline Bleeding & 0 & $12(24 \%)$ & $18(39.1 \%)$ & $30(16.2 \%)$ \\
\hline Hepatomagaly & $20(22.4 \%)$ & $37(74 \%)$ & $40(86.9 \%)$ & $(52.4 \%)$ \\
\hline Ascities & $8(8.9 \%)$ & $36(72 \%)$ & $43(93.4 \%)$ & $87(47.01 \%)$ \\
\hline Pleural effusion & $2(2.2 \%)$ & $36(72 \%)$ & $38(82.6 \%)$ & $76(41 \%)$ \\
\hline
\end{tabular}

warning signs and $24.8 \%$ as severe dengue. Pleural effusion was seen in $41 \%$ of cases. It is predominantly seen on right side and in most of the cases it was minimal to moderate. CNS manifestations were noted in $4.3 \%$ of the patients. Altered sensorium, seizures, respiratory distress were noted more common in severe dengue cases. Shock was presenting feature in $21 \%$ of cases. In many patients, bradycardia and pruritis were observed during recovery phase.

Mean $\mathrm{Hb}$ was $11.32 \mathrm{~g} \%$. It was $10.36,11.21$, and 12.93 in, Dengue fever without warning signs, Dengue fever with warning signs and Severe Dengue respectively. Mean hematocrit was 37.46. Hematocrit in Dengue with warning signs and severe Dengue was higher than Dengue without warning signs group. Thrombocytopenia was noted in $89.6 \%$ of cases.

Majority of the cases had platelet count between 50000 to 1 lakh and only $8.6 \%$ of the cases had count less than 20000 / $\mathrm{mm}^{3}$. In this study none of the patients with platelet count less than 20000 had bleeding manifestations while $32.1 \%$ had bleeding manifestation with platelet count between 20000 and 50000. Platelet count below 50,000 were found more percentage in Dengue with warning signs than severe Dengue cases but statistically it is not significant $(\mathrm{P}=.156)$.

SGPT and SGOT levels were elevated in Dengue with warning signs and severe dengue. Elevation of SGPT is statistically significant $(\mathrm{P}=.005)$. Overall LFT was abnormal in $31.9 \%$ of total patients and among these $73 \%$ had abnormal LFT in severe dengue cases which is statistically significant $(\mathrm{p}<0.001)$

In ultrasound abdomen, hepatomegaly was found in higher percentage in dengue with warning signs and in severe dengue. Gallbladder wall thickness was observed in $80.4 \%$ of patients, Ascities in $93.4 \%$ of severe dengue cases. It is statistically significant when compared with dengue without warning signs $(\mathrm{P}=0.046)$.

Gall bladder wall edema and polyserositis were significantly more in Dengue with warning signs and severe dengue with p value .007 and .002 respectively. About 38 children had no abnormalities in ultrasound abdomen. Children in dengue without warning signs had more reports of normal ultrasound abdomen when compared to other two groups.

All cases without warning signs were managed conservatively. About $46.4 \%$ cases in severe dengue were managed conservatively. Platelet transfusion was given only in $24.7 \%$ cases in patients with bleeding manifestations with platelet count $<50,000$, even without bleeding manifestations with low platelet count $(<20,000)$ as per WHO guidelines.

Whole blood was given in patients with anemia and in cases where there was rapid rise in hematocrit. Ionotropes were used in $6.7 \%$ of cases. Out of 185 children, two children from severe dengue group died. Mortality rate was $1.08 \%$ 


\section{Discussion}

Total number of 185 sero positive (Ns1Ag test or IgM and IgG antibody tests) Dengue fever cases were analyzed during our study period. According to revised Dengue classification system proposed by WHO, cases were classified as Dengue fever without warning signs (48.1\%), Dengue fever with warning signs (27\%) and severe Dengue $(24.8 \%)$ based on signs, symptoms \& lab parameters. In this study maximum number of cases belong to age group of 5 to $15 \mathrm{yrs}$ with mean age age of $8.2 \mathrm{yrs}$ and this is in accordance with other studies [7]. The incidence of male children that were affected is slightly more in our study, the ratio being 1.9:1. Similar kind of male predominance observed in other studies like Agarwal et al[8], Narayana et al[9] and Gomber et al[10]. In the present study predominantly patients were from urban areas though WHO has reported shift of dengue to rural areas. A seasonal pattern was observed in our study. Highest incidence occurred during the monsoon and post monsoon season. Wongkoon $\mathrm{S}$ et al [11] have also described seasonal pattern of dengue which corresponded with the rainy season due to abundance of mosquito breeding in the season.

Fever was noted in all patients. Vomitting was the next common symptom in our study. Agarwal, et al [8] in their study from Delhi, have also noted fever, abdominal pain and vomiting as the commonest symptoms. In one more study by wang et al [12]observed vomiting (60.5\%) and pain abdomen $(32.5 \%)$ were common presenting symptom in children whereas arthralgia and myalgia were less common symptom in children when compared to adults which was also stastically significant $(\mathrm{p}<0.001)$. Headache was seen in $32.4 \%$ in the present study. About $28.8 \%$ observed in Narayanan et al study, $77 \%$ in Kalyanarooj et al[13] study and $22 \%$ in Ratageri et al[14] study.

Malena and epistaxis were most common bleeding manifestation noted in this study and similar observation made by Ahmed et al [15] study. There was no correlation between platelet counts and bleeding manifestations. In our study too, though thrombocytopenia was found in $89.6 \%$ of cases, only $16.2 \%$ of cases had bleeding manifestations.

CNS involvement was seen in 8 patients who presented with drowsiness, altered sensorium and later developed convulsions in some cases. As the involvement of CNS system in Dengue fever is recognized more frequently, Dengue encephalopathy should be considered as a possibility in any child presenting with neurological symptoms in endemic areas.

Overall Hepatomegaly was seen in $52.4 \%$ in present study which was similar to other studies [8,9] Hepatomegaly (86.9\%), Ascities (93.4\%), pleural effusion (82.6\%), and Facial puffiness (84.7\%) were significantly high in severe dengue as compared to dengue fever without warning signs which is statistically significant $(\mathrm{p}<0.001)$. presence of these signs will help in predicting the severity of the disease as noted in other studies also.

Mean hematocrit in present study was $37.46 \%$ which was almost similar to other studies [9,14]. Mean hematocrit value was significantly higher in Dengue with warning signs and severe dengue group. Thrombocytopenia was reported in $89.6 \%$ of cases. Majority of the cases had platelet count between 50000 to 1 lakh and only $8.6 \%$ of the cases had count less than $20000 / \mathrm{mm}^{3}$ and similar observation was seen in other studies. Aggarwal et al reported, >50000 (31\%), 2500050000 (47\%), <25000 (22\%) platelet count. Presence of leucopenia, thrombocytopenia (89.6.\%) and increasing haemoconcentration gave important evidence towards diagnosis of dengue fever. Liver enzymes (SGOT and SGPT) were raised in $31.9 \%$ of cases overall and $73 \%$ had abnormal LFT in severe dengue as noted in other studies. Presence of abnormal LFT and Ascites were more significantly associated with severe dengue.

Sonographic findings of dengue fever have been described in several literatures. Some authors even conclude that during an epidemic the ultrasound findings of gall bladder wall thickening with or without polyserosities in a febrile patient should suggest the possibility of Dengue fever [16]. Gallbladder wall thickness $(80.4 \%)$ was the most common ultrasound abdomen findings noted which was similar to other studies [17]. In present study ascites, gall bladder wall edema and hepatomegaly on ultrasound were significantly more noted in severe dengue $(\mathrm{P}<0.05)$.

Other studies also reveal the same. Setiwan et al [18] and Mehdi SA et al [19] noted $95 \%$ and $60 \%$ of ascites in severe dengue and $34 \%$ and $17.7 \%$ in mild Dengue respectively. They concluded that ascites is a good indicator to predict severity of dengue fever. Serological diagnosis of dengue viral infection was done but viral isolation and PCR was not done due to 
non-availability of facility. In our study, non-structural protein 1 antigen was positive in $71 \%$ of cases indicating it is a highly sensitive test for the detection of dengue early during the illness [20]. To increase the dengue diagnostic sensitivity both NS1 Ag and IgM detection in samples during first few days is recommended. IgG is the dominant immunoglobulin in secondary dengue infection and $\operatorname{IgM}$ levels may not be detectable during secondary dengue infection as the levels will significantly decrease later.

In the present study $68 \%$ of dengue children required crystalloids. Colloids were not used in any patients and by adopting the revised WHO classification the need for blood products were limited to few cases.

WHO in their recent guidelines on management of dengue, has described stepwise approach to the management of dengue, where only isotonic solutions have been advised, followed by serial monitoring of clinical status, fluid balance and hematocrit. Judicious use of fluid was advised to maintain effective circulation during the leak period. Mortality in this study was $1.08 \%$. Mortality rate varies from 0 to $12 \%$ from various studies [21, 22]. Over period of time Mortality rate is drastically reduced by early recognition, precise assessment and appropriate fluid management as per WHO protocol.

\section{Conclusion}

Dengue fever is becoming more prevalent in India. Incidence of severe Dengue is increasing. Children between 5 and 15 yrs were most commonly affected by Dengue fever. Fever, Vomiting and pain abdomen were most common presenting symptom.

Hepatomegaly, ascites, pleural effusion, gall bladder wall thickening and abnormal liver function tests were found significantly in severe dengue cases which can be used as supportive markers of severe dengue. Early recognition, precise assessment and appropriate treatment with the help of WHO revised classification and management guidelines have reduced the mortality due to dengue fever.

Funding: Nil, Conflict of interest: None initiated, Perission from IRB: Yes

\section{References}

1. WHO. DENGUE. Guidelines for Diagnosis, Treatment, Prevention and Control. 2009 Geneva, World Health Organization, 2009.
2. World Health Organisation. Prevention and control of dengue and dengue haemorrhagic fever: comprehensive guidelines. WHO Regional publication, SEARO, No 29, 1999.

3. Guzmán MG, Kourí G. Dengue: an update. Lancet Infect Dis. 2002 Jan; 2 (1):33-42.

4. WHO: Dengue Guildelines for Diagnosis, Prevention and Control. New edition. Geneva, Switzerland: World Health Organisation; 2009.

5. Barniol J, Gaczkowski R, Barbato EV, da Cunha RV, Salgado D, Martínez E, Segarra CS, Pleites Sandoval EB, Mishra A, Laksono IS, Lum LC, Martínez JG, Núnez A, Balsameda A, Allende I, Ramírez G, Dimaano E, Thomacheck K, Akbar NA, Ooi EE, Villegas E, Hien TT, Farrar J, Horstick O, Kroeger A, Jaenisch T. Usefulness and applicability of the revised dengue case classification by disease: multi-centre study in 18 countries. BMC Infect Dis. 2011 Apr 21; 11:106. doi: 10.1186/1471-2334-11-106.

6. Narvaez F, Gutierrez G, Pérez MA, Elizondo D, Nuñez A, Balmaseda A, Harris E. Evaluation of the traditional and revised WHO classifications of Dengue disease severity. PLoS Negl Trop Dis. 2011 Nov; 5 (11):e1397. doi: 10.1371/journal.pntd.0001397. Epub 2011 Nov 8.

7. Mittal H, Faridi MM, Arora SK, Patil R. Clinicohematological profile and platelet trends in children with dengue during 2010 epidemic in north India. Indian J Pediatr. 2012 Apr;79(4):467-71. doi: 10. 1007/s12098-011-0586-7. Epub 2011 Oct 29.

8. Agarwal R, Kapoor S, Nagar R, Misra A, Tandon R, Mathur A, et al. A clinical study of the patients with dengue hemorrhagic fever during the epidemic of 1996 at Lucknow, India. Southeast Asian J Trop Med Public Health 1999 Dec; 30(4) : 735-40

9. Narayanan M, Aravind MA, Thilothammal N, Prema R, Sargunam CS, Ramamurty N. Dengue fever epidemic in Chennai--a study of clinical profile and outcome. Indian Pediatr. 2002 Nov;39(11):1027-33.

10. Gomber S, Ramachandran VG, Kumar S, Agarwal KN, Gupta P, Gupta P, Dewan DK. Hematological observations as diagnostic markers in dengue hemorrhagic fever--a reappraisal. Indian Pediatr. 2001 May; 38(5):477-81. 
11. Wongkoon S, Jaroensutasinee, M, Jaroensutasinee K. Distribution, seasonal variation and dengue transmission prediction in Sisaket, Thailand. Indian $\mathrm{J}$ Med Res. 2013 Sep;138(3):347-53.

12. Wang CC, Lee IK, Su MC, Lin HI, Huang YC, Liu $\mathrm{SF}$, et al. Differences in clinical and laboratory characteristics and disease severity between children and adults with dengue virus infection in Taiwan, 2002. Trans R Soc Trop Med Hyg. 2009 Sep;103 (9): 871-7.

13. Kalayanarooj S, Chansiriwongs V, Nimmannitya S. Dengue patients at the Children's Hospital, Bangkok: 1995-1999. Review Dengue Bulletin 2002;26:33-43.

14. Ratageri VH, Shepur TA, Wari PK, Chavan SC, Mujahid IB, Yergolkar PN. Clinical profile and outcome of Dengue fever cases. Indian J Pediatr. 2005 Aug; 72(8):705-6.

15. Ahmed S, Arif F, Yahya Y, Rehman A, Abbas K, Ashraf S, Akram DS. Dengue fever outbreak in Karachi 2006--a study of profile and outcome of children under 15 years of age. J Pak Med Assoc. 2008 Jan;58(1):4-8.

16. Venkata Sai PM, Dev B, Krishnan R. Role of ultrasound in dengue fever. Br J Radiol. 2005 May; 78(929):416-8.

17. Colbert JA, Gordon A, Roxelin R, Silva S, Silva J, Rocha C, Harris E. Ultrasound measurement of gallbladder wall thickening as a diagnostic test and prognostic indicator for severe dengue in pediatric patients. Pediatr Infect Dis J. 2007 Sep;26(9):850-2.

18. Setiawan MW, Samsi TK, Wulur H, Sugianto D, Pool TN. Dengue haemorrhagic fever: ultrasound as an aid to predict the severity of the disease. Pediatr Radiol. 1998 Jan; 28(1):1-4.

19. Mehdi S A, Mahais H A, Bhukhari H, Aslam S. Grey scale trans-Abdomino Thoracic Ultrasonography in evaluation of dengue hemorrhagic fever. APMC. $2012 ; 6: 32-6$

20. Kumarasamy V, Wahab AH, Chua SK, Hassan Z, Chem YK, Mohamad M, Chua KB. Evaluation of a commercial dengue NS1 antigen-capture ELISA for laboratory diagnosis of acute dengue virus infection. J Virol Methods. 2007 Mar;140(1-2):75-9. Epub 2006 Nov 30.

21. Kabra SK, Jain Y, Pandey RM, Madhulika, Singhal T, Tripathi P, Broor S, Seth P, Seth V. Dengue haemorrhagic fever in children in the 1996 Delhi epidemic. Trans R Soc Trop Med Hyg. 1999 May-Jun; 93(3):294-8.

22. Srivastava VK, Suri S, Bhasin A, Srivastava L, Bharadwaj M. An epidemic of Dengue hemorrhagic fever and Dengue shock syndrome in Delhi: a clinical study. Ann Trop Paediatr. 1990 ;10(4): 329-34.

\section{How to cite this article?}

Srinivasa K, Ajay J, Manjunath G.A. Clinical profile and outcome of dengue among hospitalized children - a single centre prospective study. J PediatrRes.2017;4(02):143-148.doi:10.17511/ijpr.2017.i02.10. 